CLINICAL STUDY

\title{
Incidence of second neoplasm in childhood cancer survivors treated with GH: an analysis of GeNeSIS and HypoCCS
}

Whitney W Woodmansee, Alan G Zimmermann ${ }^{1}$, Christopher J Child ${ }^{2}$, Qi Rong ${ }^{1}$, Eva Marie Erfurth ${ }^{3}$, Paolo Beck-Peccoz ${ }^{4}$, Werner F Blum ${ }^{5}$, Leslie L Robison ${ }^{6}$ on behalf of the GeNeSIS and HypoCCS International Advisory Boards

Harvard Medical School, Brigham and Women's Hospital, 221 Longwood Avenue, Boston, Massachusetts 02115, USA, ${ }^{1}$ Lilly Research Laboratories, Indianapolis, Indiana, USA, ${ }^{2}$ Lilly Research Laboratories, Windlesham, UK, ${ }^{3}$ Skånes University Hospital, Lund University, Lund, Sweden, ${ }^{4}$ Fondazione IRCCS Cà Granda Policlinico, University of Milan, Milan, Italy, ${ }^{5}$ Lilly Research Laboratories, Bad Homburg, Germany and ${ }^{6}$ St Jude Children's Research Hospital, Memphis, Tennessee, USA

(Correspondence should be addressed to WW Woodmansee; Email: wwoodmansee@partners.org)

\begin{abstract}
Objective: Childhood cancer survivors are commonly treated with GH for GH deficiency that develops either as a result of primary malignancy or its treatment. One study - the Childhood Cancer Survivor Study (CCSS) - demonstrated increased risk of second neoplasm (SN) in GH-treated childhood cancer survivors compared with non-GH treated, after adjusting for key risk factors. We assessed the incidence of SN in GH-treated childhood cancer survivors in outpatient observational studies of GH replacement. Design: Retrospective analysis of two prospective cohort studies that collected data on safety of GH replacement as prescribed in clinical practice.

Methods: Childhood cancer survivors enrolled in Eli Lilly and Company's pediatric (Genetics and Neuroendocrinology of Short Stature International Study (GeNeSIS)) and adult (Hypopituitary Control and Complications Study (HypoCCS)) observational studies of GH treatment were assessed for incidence of SN.

Results: The percentage of childhood cancer survivors treated with GH who developed a SN was 3.8\% in pediatric GeNeSIS participants and $6.0 \%$ in adult HypoCCS participants. The estimated cumulative incidence of SN at 5 years of follow-up in these studies was 6.2 and $4.8 \%$ respectively.

Conclusions: The incidence of SN in GeNeSIS and HypoCCS GH-treated participants is similar to the published literature and is thus consistent with increased risk of $\mathrm{SN}$ in childhood cancer survivors treated with GH. As follow-up times were relatively short ( $<3$ years), longer observation is recommended. Nevertheless, clinicians should be alerted to the possibility of increased risk of $\mathrm{SN}$ in childhood cancer survivors treated with GH and continue chronic surveillance.
\end{abstract}

European Journal of Endocrinology 168 565-573

\section{Introduction}

GH deficiency (GHD) is an endocrinopathy found in childhood cancer survivors, resulting from tumors affecting the hypothalamic-pituitary axis or from cancer therapy, especially cranial irradiation $(1,2,3)$. For example, GHD has been found in 58\% of long-term survivors of childhood acute lymphoblastic leukemia treated with $\geq 24$ Gy whole brain radiation (4). Although GH replacement therapy is often used to stimulate growth in these patients, there have been concerns regarding long-term safety. Preclinical studies have demonstrated that both $\mathrm{GH}$ and insulin-like growth factor 1 (IGF1) have mitogenic properties, stimulating cellular proliferation and inhibiting apoptosis in a variety of in vitro assays and possibly being tumorigenic in animals under certain conditions $(5,6)$. In humans, epidemiological studies have shown a positive correlation between IGF1 blood levels and the risk of certain tumors (colorectal, prostate, and breast $(7,8,9))$. Despite these associations, increased risk of de novo or recurrent malignancy in GH-deficient patients treated with $\mathrm{GH}$ has not been confirmed $(10,11,12,13,14,15,16,17)$.

Two analyses from a single database, the Childhood Cancer Survivor Study (CCSS) $(18,19)$, have reported that GH treatment is associated with an increase in the relative risk of second neoplasm (SN) in childhood cancer survivors. Exposure to radiation and alkylating agents were identified as additional risk factors for the development of SNs. As a result of these findings, the Food and Drug Administration in the USA requested in 2006 that all GH product labels should be strengthened to reflect increased risk of $\mathrm{SN}$ in childhood cancer survivors. 
The aim of the current analysis was to assess the risk of SN in childhood cancer survivors enrolled in Eli Lilly and Company's pediatric (Genetics and Neuroendocrinology of Short Stature International Study (GeNeSIS)) and adult (Hypopituitary Control and Complications Study (HypoCCS)) observational studies of $\mathrm{GH}$ treatment. To our knowledge, this is the first analysis to examine the risk of $\mathrm{SN}$ in childhood cancer survivors enrolled in large postmarketing observational studies of GH-treated individuals.

\section{Materials and methods}

\section{Study databases}

GeNeSIS (ClinicalTrials.gov, NCT01088412) is an international, open-label postmarketing surveillance program (prospective cohort study) established in 1999. The aim of GeNeSIS is to collect information on the clinical management and treatment outcomes of pediatric patients with short stature, growth disorders, or disorders of hypothalamic-pituitary function who are treated, at the discretion of the participating physician, with GH (Humatrope; somatropin, Eli Lilly and Company), according to standard pediatric endocrinology practice. The current analysis is based on data included in the February 2008 database lock (data to September 2007). At that time, the database included 11258 participants who had at least one follow-up visit recorded and were eligible for analysis; of these, 11136 had received at least one GH dose during GeNeSIS participation (GH-treated group) and 122 had no reported $\mathrm{GH}$ therapy.

HypoCCS (ClinicalTrials.gov, NCT01088399) is a postmarketing surveillance program (prospective cohort study) with a primary objective to determine whether long-term GH replacement therapy in GH-deficient adults is associated with a changed incidence of clinically significant adverse events. All participants in HypoCCS had an established diagnosis of adult GHD, either alone or combined with other pituitary hormone deficiencies, as determined by clinical history and/or biochemical testing (20). The diagnostic approach used and the decision to administer $\mathrm{GH}$ was at the discretion of the investigating physician. Patients were considered ineligible for HypoCCS enrollment if they had unresolved or unstable conditions listed as contraindications or precautions for GH therapy. Such conditions include evidence or suspicion of active malignancy or evidence of ongoing pituitary or other intracranial tumor activity. Patients from North America and Europe were originally enrolled in separate studies. In 2002, a global protocol was initiated, and by 2005 , all data were merged into a single database. The current analysis was based on the merged HypoCCS database of October 2008 (data to July 2008). At that time, the HypoCCS database included 7785 hypopituitary participants who had at least one follow-up visit recorded; of these, 6840 had received at least one GH dose during HypoCCS participation (GH-treated group), 940 had no reported GH therapy during HypoCCS participation, and five were of unknown treatment status (excluded from analysis). Of the $6840 \mathrm{GH}$-treated participants, 5522 $(80.7 \%)$ were reported as having adult-onset GHD and $1299(19.0 \%)$ as having childhood-onset GHD (onset type not reported for 19 participants).

Both HypoCCS and GeNeSIS are conducted in accordance with the Declaration of Helsinki and adhere to applicable regulatory requirements in the participating countries. Institutional review board approval and written informed consent from participants, parents, or legal guardians for data collection, electronic processing, and publication were obtained in accordance with national laws. Study data were collected as provided by the study investigator and were cross-referenced with the Lilly Safety System (LSS) pharmacovigilance database to confirm accuracy or obtain additional details. LSS is a separate database that collects detailed information on serious adverse events for all company products (for studies such as HypoCCS and GeNeSIS, this information is provided by the study investigator). The participants for the current HypoCCS analysis were recruited from 16 countries: Austria, Belgium, Canada, Czech Republic, Denmark, France, Germany, Hungary, Iceland, Italy, Norway, Spain, Sweden, The Netherlands, UK, and the USA. The participants for the GeNeSIS analysis were recruited from these 16 countries plus 14 additional countries: Australia, Finland, Greece, India, Japan, Kazakhstan, Lithuania, Pakistan, Russia, Singapore, Slovakia, South Africa, Taiwan, and Thailand.

\section{Definition of childhood cancer survivor}

Only childhood cancer survivors, defined as participants whose initial cancer diagnosis occurred at age $<21$ years, were eligible for inclusion in these analyses. For GeNeSIS, identification of this cohort was based on data collected at study entry, which included information regarding diagnosis of GHD and previous neoplastic conditions. Additional information on previous neoplastic conditions was ascertained from reports of SNs as serious adverse events in the LSS pharmacovigilance database. A total of 491 childhood cancer survivors were identified in GeNeSIS, of whom 70 were excluded because no follow-up data were available (Fig. 1A). Thus, 421 GeNeSIS childhood cancer survivors were included in our analysis. For HypoCCS, identification of the childhood cancer survivors was more challenging because the current global database is a merger of three similar, but not identical, protocols. Consequently, we used broad search criteria to capture all potential childhood cancer survivors in the database and included all data collection modules that might provide pertinent information. Further in-depth review by two study physicians confirmed that 310 participants met eligibility criteria; 
A

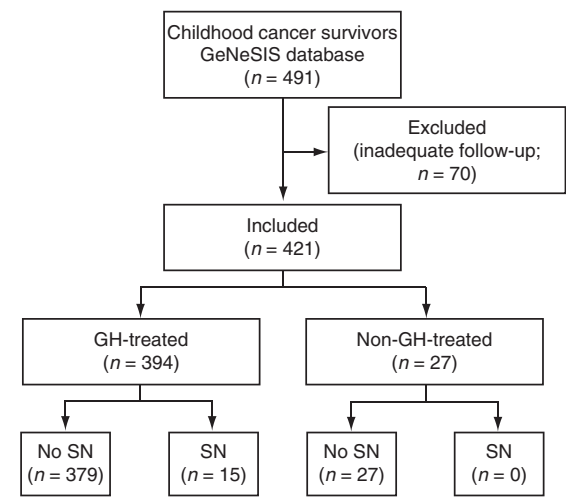

B

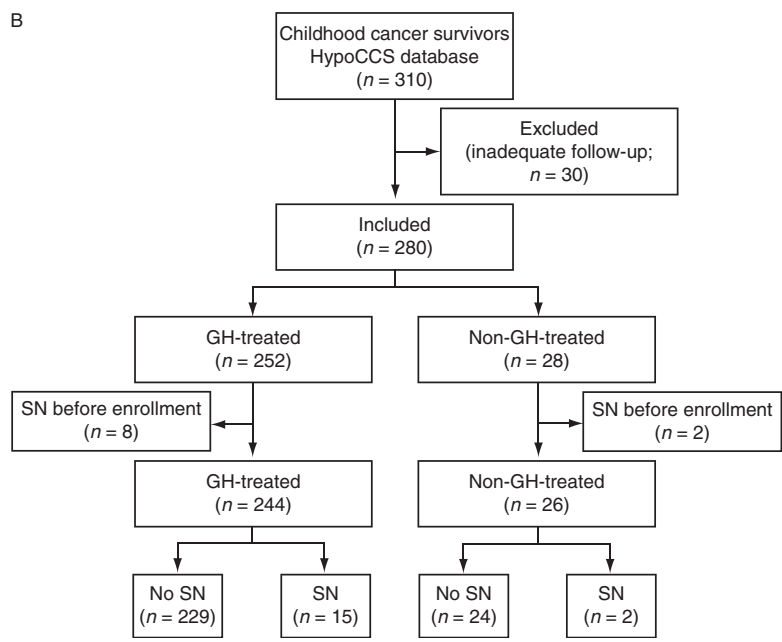

Figure 1 Flow diagram of patients enrolled in the GeNeSIS (A) and HypoCCS (B) programs who were included in this analysis. SN, second neoplasm.

30 of these were excluded from the analysis because of missing follow-up data (Fig. 1B).

\section{Identification of SN cases among childhood cancer survivors}

Study adverse event records were reviewed to ascertain SN cases. A SN was defined as a benign or malignant neoplasm following a previous childhood cancer, but excluding metastases or recurrence of the primary cancer. A SN may have a different histological type and may occur in the same or different organs as the previous neoplasm, in all cases arising from an independent oncogenic event.

The criteria for determination of benign and malignant primary neoplasms and SNs, from the diagnoses provided by study investigators, were identical for both GeNeSIS and HypoCCS. In cases where tumor categorization was uncertain, the following rules were applied: i) pituitary tumors and craniopharyngiomas were considered benign, unless specifically denoted as malignant; ii) meningiomas of low or unspecified grade were classified as benign because transition to malignancy is rare; iii) cases of histiocytosis, neurofibromatosis, and teratoma were considered benign, unless specifically denoted as malignant; iv) gliomas and astrocytomas were considered malignant, unless specifically described as low grade or pilocytic, or as pleomorphic xanthoastrocytomas (per World Health Organization criteria (21), these specific tumor types were considered benign, although transition into malignancy is possible); v) pineal region neoplasms for which malignancy was not specified were considered malignant; vi) cases where available information indicated total body irradiation were considered to have had a previous malignancy; and vii) ambiguous cases where the tumor type was not specified were not considered as malignancies.

\section{IGF1 concentrations}

Where possible, serum IGF1 concentrations were measured centrally using a RIA (University of Giessen, Germany in Europe and Esoterix Endocrinology, Calabasas Hills, CA, USA), and IGF1 SDS were calculated as described previously $(22,23)$. Additionally, in GeNeSIS, a number of IGF1 concentrations were determined by local laboratories and converted to central laboratory values by cross-calibration of the assays used (24).

\section{Statistical analysis}

As GeNeSIS and HypoCCS are separate studies with distinct protocols and data collection methods, data from each study were analyzed separately. Demographic data were summarized as median and first (Q1) and third (Q3) quartiles, and the proportion of patients with a SN was calculated with $95 \%$ CIs for both study populations. Cumulative incidence (with s.E.M.) of SN was estimated using death as a competing risk, adjusting for varying follow-up times per patient and censored data for those patients without a SN or death (25). If there were no competing risk events (deaths), the methodology is equivalent to using a Kaplan-Meier curve to estimate the percentage of patients without a SN at a given time point and subtracting the KaplanMeier estimate from 1. For GeNeSIS, the cumulative incidence of SN was calculated from the start date of GH therapy. For HypoCCS, as the GH start date was not always known for participants who started GH therapy before study entry, the cumulative incidence of SN was calculated from the date of enrollment into HypoCCS. Finally, because of baseline demographic differences between GH-treated and non-GH-treated participants, and lack of other key information that should be adjusted for in a formal comparison (e.g. radiotherapy and chemotherapeutic agent dose information), a formal statistical comparison between treatment groups was not considered appropriate. 


\section{Results}

\section{GeNeSIS analysis}

Demographics In GeNeSIS, 491 childhood cancer survivors were identified, of which 421 had at least one follow-up visit and were included in the analysis (Fig. 1A). The most common primary cancers were medulloblastoma $(n=140,33.3 \%)$ and leukemia $(n=63,15.0 \%)$. Among the 394 (232 male and 162 female) GH-treated childhood cancer survivors, the age of primary cancer diagnosis was documented in 352 , with a median $(Q 1, Q 3)$ age of $5.4(3.0,8.5)$ years. Median age at the start of GH therapy was 10.8 $(8.9,12.9)$ years and median duration of treatment was $2.9(1.4,4.8)$ years. Among the 27 (19 males and eight females) non-GH-treated childhood cancer survivors, 26 had the age of primary cancer diagnosis documented, with a median of $7.5(3.6,10.3)$ years. Overall, the median time enrolled in GeNeSIS was 2.1 $(1.1,3.5)$ years.

Incidence of SN There were 15 cases of SN identified among the 394 GH-treated participants (Fig. 1A, Table 1), corresponding to a calculated SN proportion of 3.8\% (95\% CI, 2.2-6.2\%), and no cases of SN among the 27 non-GH-treated participants. SN followed medulloblastoma in 10 of 15 cases (Table 1). The most common SN reported was meningioma (three cases) and all followed therapy for medulloblastoma. All but one case of SN occurred in participants who had received both chemotherapy and radiation exposure as part of their primary cancer treatment. The median time (Q1, Q3) from primary cancer diagnosis to development of SN was $8.4(6.3,10.6)$ years and the median time from start of GH therapy to SN was 2.4 $(1.6,4.4)$ years. The cumulative incidence of SN from the start of $\mathrm{GH}$ treatment was estimated for the 394 GH-treated childhood cancer survivors (Fig. 2) and was $6.2 \%$ (s.E.M., $1.9 \%$ ) at 5 years of follow-up. Seven deaths were reported in this population of 394 GH-treated participants during the follow-up period. Three deaths were associated with SN (Table 1) - neuroblastoma following acute lymphocytic leukemia, acute myeloid leukemia following medulloblastoma, and myelodysplastic syndrome following medulloblastoma (with death due to complications associated with graft vs host disease after bone marrow transplant). Three deaths were due to recurrences of primary cancers (astrocytoma, medulloblastoma, and neuroblastoma), and the remaining case due to a road traffic accident. Additionally, there was one death among the 27 non$\mathrm{GH}$-treated patients (medulloblastoma recurrence).

GH dose and IGF1 SDS The mean GH dose after 1 year of treatment was 0.22 (s.E.M., 0.03) $\mathrm{mg} / \mathrm{kg}$ per week in the GH-treated participants who developed a $\mathrm{SN}$ and 0.24 (s.E.M., 0.01) $\mathrm{mg} / \mathrm{kg}$ per week in those who did not. Mean baseline IGF1 SDS was -1.7 (s.E.M., 0.66) in those who developed a SN and - 3.2 (s.E.M., 0.23) in those who did not. Both groups of GH-treated participants had improved mean IGF1 SDS after initiation of GH therapy (Fig. 3A).

Table 1 Childhood cancer survivors in GeNeSIS who developed a second neoplasm.

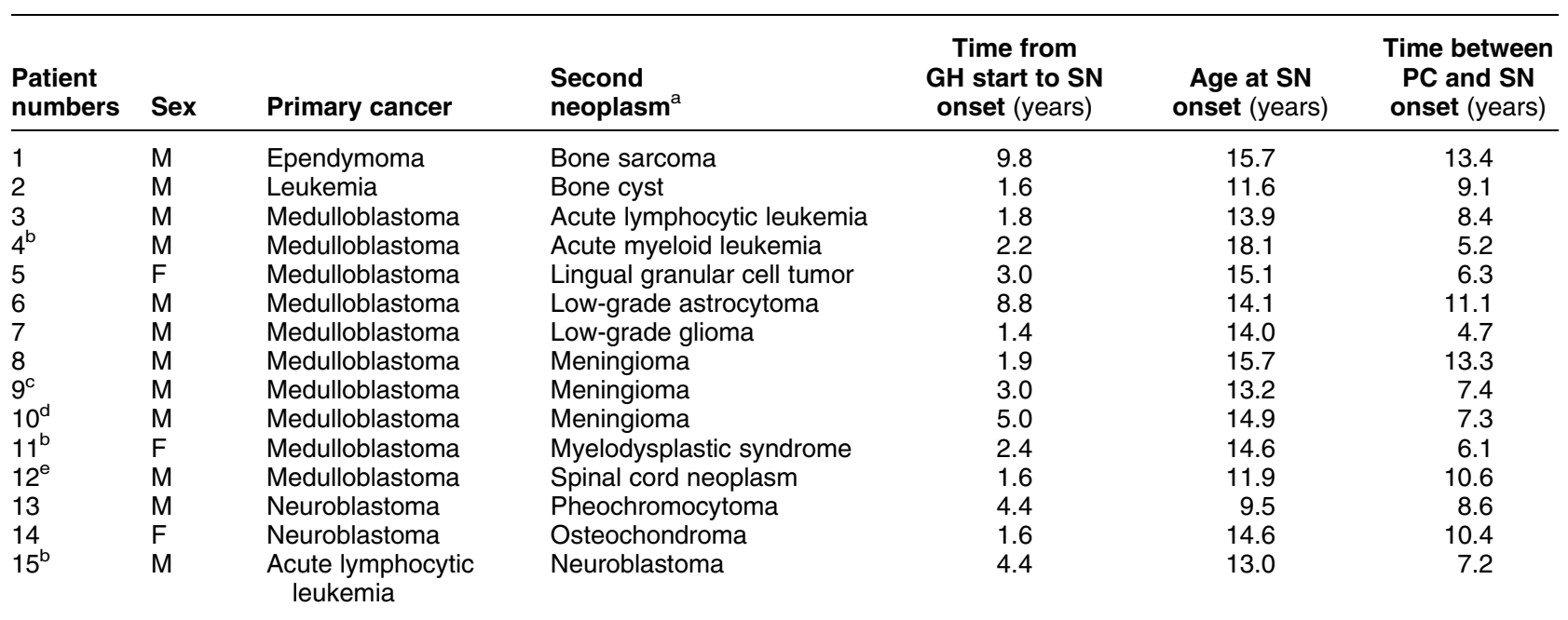

F, female; M, male; PC, primary cancer; SN, second neoplasm.

${ }^{a}$ All childhood cancer survivors in the GeNeSIS who developed a second neoplasm were treated with GH. All participants had a history of radiation therapy as part of treatment for their primary cancer.

${ }^{\mathrm{b}}$ Patient died with cause of death associated with second neoplasm.

${ }^{\mathrm{c}}$ This patient had a history of Gorlin syndrome and had additional second neoplasms (basal cell carcinoma and benign soft tissue lesion).

${ }^{d}$ All patients had been treated with chemotherapy for their primary cancer except this patient.

${ }^{\mathrm{e}}$ The second neoplasm in this patient was not confirmed by repeated magnetic resonance imaging. 


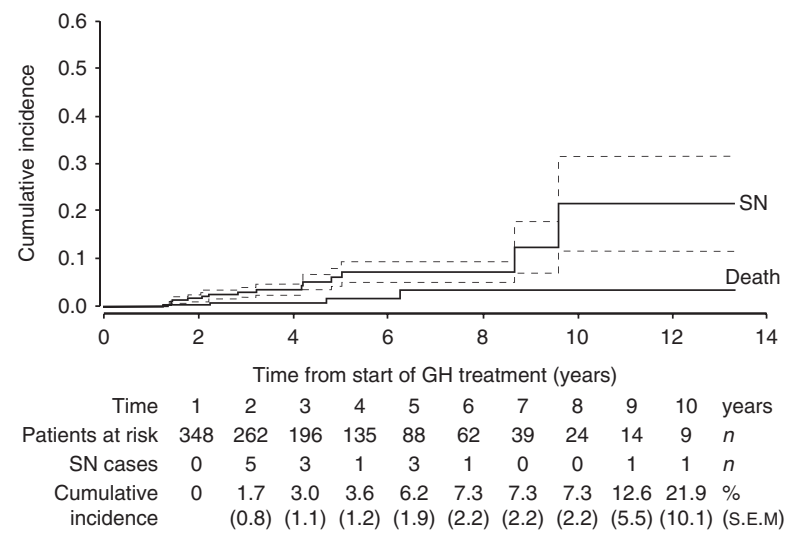

Figure 2 Cumulative incidence curve for incidence of second neoplasm (SN) or death (not associated with SN) in GH-treated childhood cancer survivors enrolled in GeNeSIS. The cumulative incidence of $\mathrm{SN}$ is shown by the upper solid line with the cumulative incidence \pm 1 S.E.M. indicated by the dashed lines. The cumulative incidence of death is shown by the lower solid line but does not include three cases where death was associated with SN. The number of patients at risk at year 0 was 394.

\section{HypoCCS analysis}

Demographics A total of 310 participants were confirmed as childhood cancer survivors, of whom 280 (252 GH-treated and 28 non-GH-treated) had a follow-up visit recorded and were included in the analyses (Fig. 1B). In the 280 participants overall, the most common primary cancer diagnoses were germinoma $(n=60,21.4 \%)$, leukemia $(n=51,18.2 \%)$, medulloblastoma $(n=44,15.7 \%)$, and astrocytoma $(n=44, \quad 15.7 \%)$. Of the 252 (117 males and 135 females) GH-treated childhood cancer survivors, the age of primary cancer diagnosis was documented in 107 participants, with a median age (Q1, Q3) of 8.4 years $(4.1,12.2)$. Among all $252 \mathrm{GH}$-treated participants, the median duration of GHD at study entry was 6.8 years $(0.8,14.6)$ and the duration of follow-up in HypoCCS was 2.9 years $(1.5,5.1)$. Of the 28 non-GH-treated participants, only seven had the age at primary cancer diagnosis recorded, with a median age of 8.7 years $(4.1,13.6)$. Among all 28 non$\mathrm{GH}$-treated participants, the median duration of GHD at study entry was 13.8 years $(5.1,20.1)$ and median duration of study follow-up was 2.6 years $(1.8,3.7)$.

Incidence of $\boldsymbol{S N}$ After case review, a total of 27 (23 GH-treated and four non-GH-treated) SNs were identified in 280 childhood cancer survivors, ten of which occurred before enrollment in HypoCCS (Fig. 1B, Table 2). Of these ten cases, four were naïve to $\mathrm{GH}$ therapy at the time of enrollment and six had been treated previously with $\mathrm{GH}$; the timing of SN development relative to previous GH therapy in these cases was unknown. The SN proportion during HypoCCS was $6.0 \%(95 \%$ CI, $3.4-9.6 \%)$ based on 15 cases in 252
GH-treated childhood cancer survivors and 7.1\% (95\% CI, 0.9-23.5\%) based on two cases in 28 patients with no GH treatment. As with GeNeSIS, the most common SN was meningioma (10 of $27 ; 37.0 \%$ ). Among all childhood cancer survivors who developed a SN and had a known exact event date, the median time $(\mathrm{Q} 1, \mathrm{Q} 3)$ between primary cancer diagnosis and SN was 20.3 years $(18.1,28.9 ; n=14)$ in $\mathrm{GH}$-treated participants and 29.5 years $(10.8,31.2 ; n=3)$ in non-GH-treated participants. The estimated cumulative incidence of SN at 5 years of follow-up was $4.8 \%$ (s.E.M., $1.6 \%$ ) in GH-treated participants (Fig. 4). For the purposes of this analysis, the ten cases of SN that occurred before study enrollment were included in the group without a SN. Although none of these ten cases reported a new third neoplasm during HypoCCS participation, two cases did report recurrence of the $\mathrm{SN}$ (both meningiomas; Table 2). A total of four deaths were reported in the overall population of 280 childhood cancer survivors during the follow-up period. Three deaths were in $\mathrm{GH}$-treated participants, one of which was due to a $\mathrm{SN}$ (glioblastoma following medulloblastoma; Table 2). The cause of death was not reported in one GH-treated patient with history of medulloblastoma and in a patient with history of germinoma was due to acute
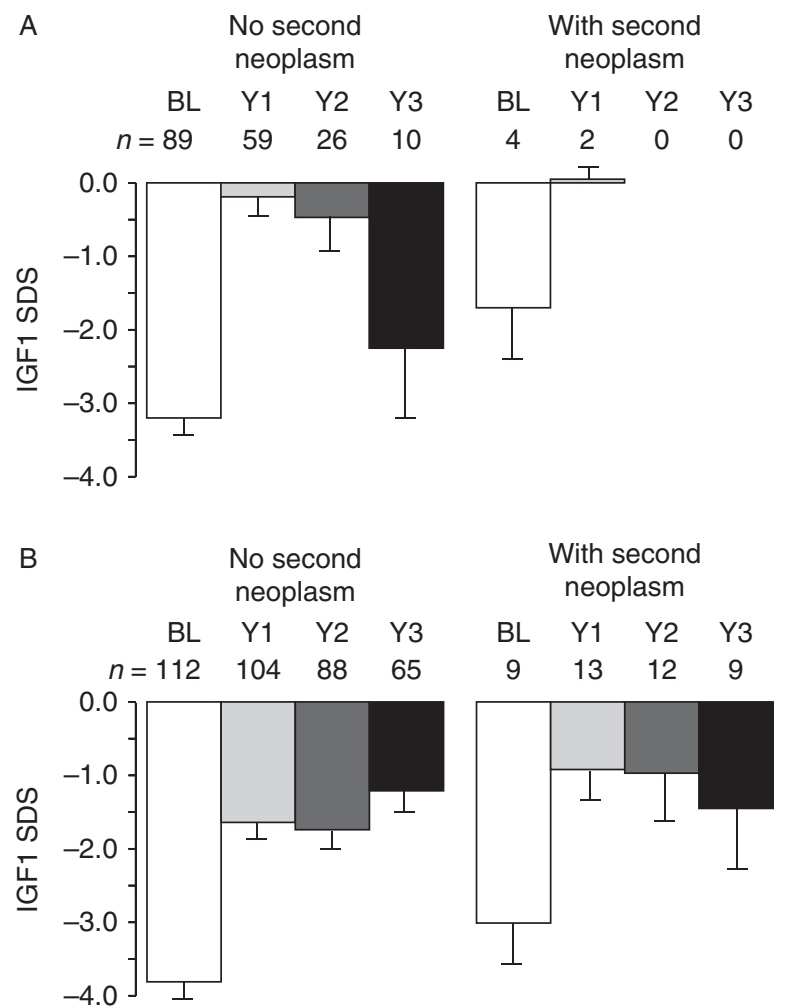

Figure 3 Insulin-like growth factor 1 (IGF1) SDS in GH-treated childhood cancer survivors enrolled in GeNeSIS (A) and HypoCCS (B). Shown are mean (+ S.E.M.) IGF1 SDS at baseline (BL; time of enrollment) and at years 1, 2, and 3 (Y1, Y2, and $Y 3$ ) for survivors with and without a second neoplasm. 


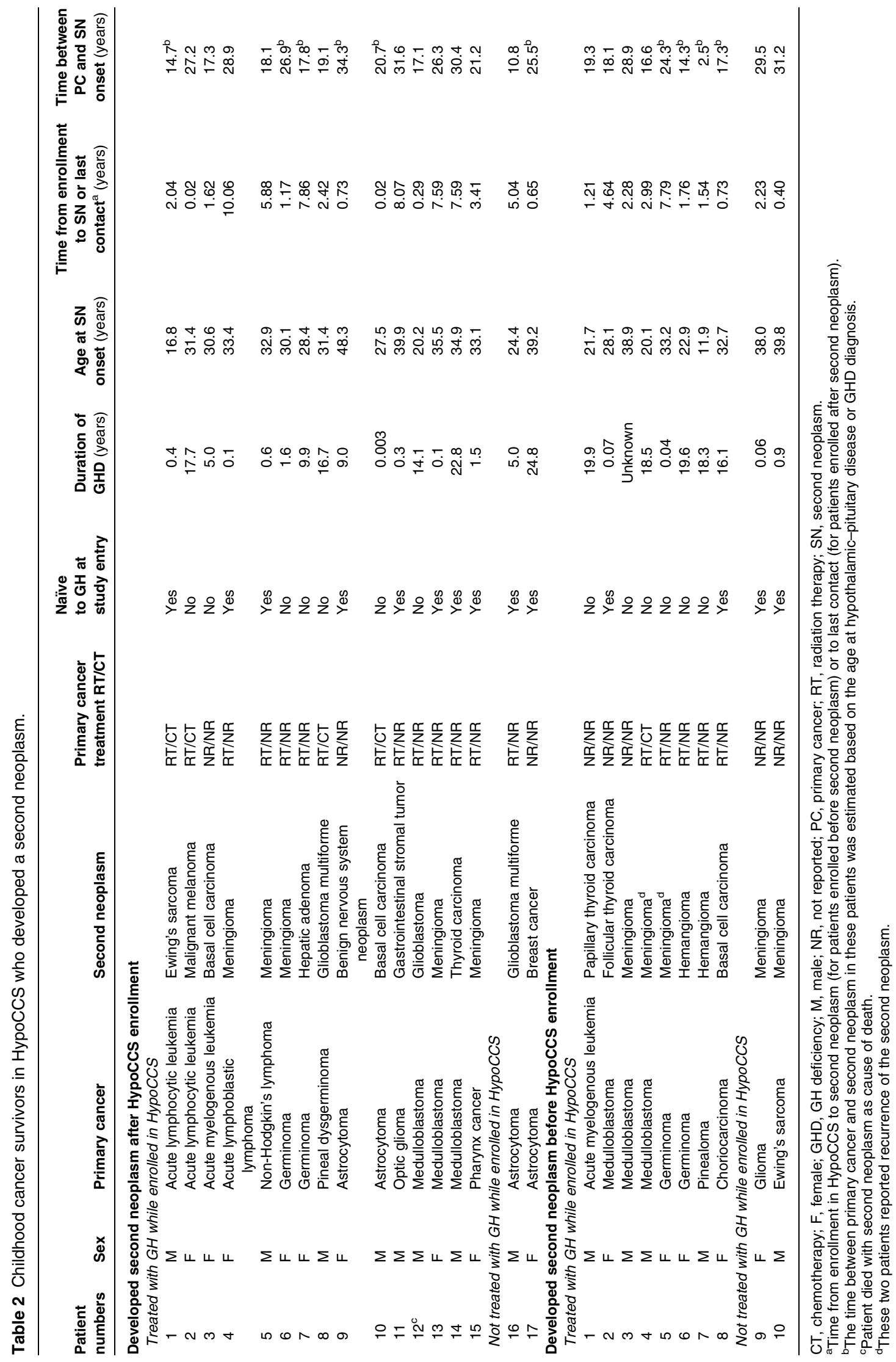




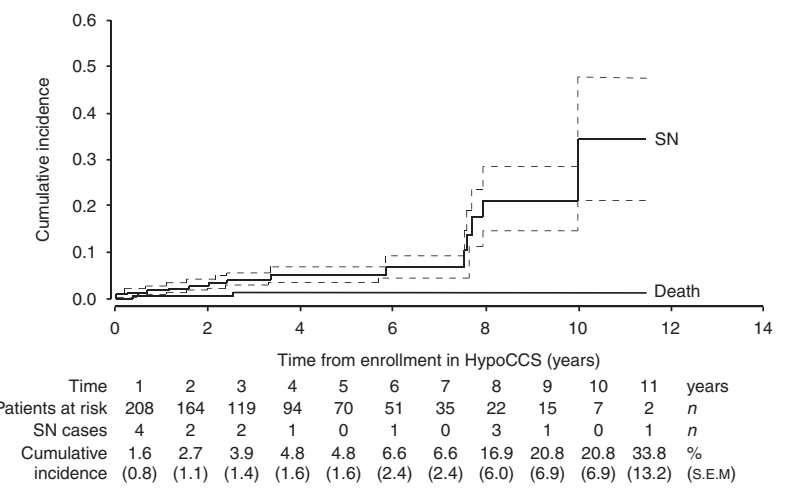

Figure 4 Cumulative incidence curve for incidence of second neoplasm (SN) or death (not associated with SN) in GH-treated childhood cancer survivors enrolled in HypoCCS. The cumulative incidence of $\mathrm{SN}$ is shown by the upper solid line with cumulative incidence \pm 1 S.E.M. indicated by the dashed lines. The cumulative incidence of death is shown by the lower solid line but does not include one case where death was associated with SN. The number of patients at risk at year 0 was 252 .

fulminant pancreatitis. One non-GH-treated patient with a history of germinoma died of 'hepatic coma'.

GH dose and IGF1 SDS Mean GH dose was calculated annually for the first 3 years of treatment for GH-treated participants stratified by whether or not they developed a SN. In participants with a SN, mean $\mathrm{GH}$ dose was 0.472 (s.E.M., 0.071) $\mathrm{mg} /$ day in year 1 , 0.554 (s.E.M., 0.098) $\mathrm{mg} /$ day in year 2, and 0.517 (S.E.M., 0.109) $\mathrm{mg} /$ day in year 3 . In participants without a SN, mean GH dose was 0.500 (s.E.M., 0.022) $\mathrm{mg} /$ day in year $1,0.525$ (s.E.M., 0.032) $\mathrm{mg} /$ day in year 2 , and 0.515 (s.e.M., 0.029) $\mathrm{mg} /$ day in year 3. The mean baseline IGF1 SDS was - 3.0 (S.E.M., 0.56) in those who developed a SN and -3.8 (s.E.M., 0.22) in those who did not. Both groups of GH-treated participants had improved IGF1 SDS after initiation of GH therapy (Fig. 3B).

\section{Discussion}

To our knowledge, this is the first report to assess the incidence of SN in childhood cancer survivors enrolled in large, observational studies of GH treatment of both pediatric and adult patients. The percentage of GH-treated childhood cancer survivors who developed a $\mathrm{SN}$ was $3.8 \%$ in pediatric GeNeSIS participants and $6.0 \%$ in adult HypoCCS participants. The estimated cumulative incidence of SN at 5 years of follow-up in these studies was 6.2 and $4.8 \%$ respectively.

Childhood cancer survivors are at increased risk of second malignancies - the Surveillance Epidemiology and End Results (SEER) program showed a sixfold increased risk of subsequent malignancy compared with the general population (26). Our patient population is not directly comparable because subjects had childhood malignancies that resulted in GHD. Our study population is enriched with patients with the known second malignancy risk factors of CNS malignancy and radiation exposure $(26)$.

The results of our analysis are consistent with previously published data from the CCSS $(18,19)$. In the initial CCSS report (19), the percentage of $\mathrm{GH}$-treated childhood cancer survivors who developed a SN during a median of 6.2 years of follow-up was $4.2 \%$ (15 SNs in 361 GH-treated childhood cancer survivors). The relative risk (adjusted) of $\mathrm{SN}$ in $\mathrm{GH}$-treated vs non-GH-treated participants was 3.21 (95\% CI, 1.9-5.5; $P<0.0001)$. After an additional 32 months of follow-up, the relative risk of $\mathrm{SN}$ in the same cohort of GH-treated vs non-GH-treated participants was 2.15 (95\% CI, 1.3-3.5; P<0.002) (18). Our results are consistent in terms of the proportion of patients with SN observed in GH-treated childhood cancer survivors and support the reproducibility of the CCSS data in two separate large patient cohorts (GeNeSIS and HypoCCS). However, there are some notable differences between our studies and the CCSS. Unlike the retrospective cohort CCSS, our studies only prospectively followed GH-treated patients. Additionally, the duration of follow-up was much longer in the CCSS and the incidence of $\mathrm{SN}$ is known to increase with longer periods of observation (27).

Our findings are also similar to those of a recent retrospective study by Mackenzie et al. (28), in which $4.5 \%$ ( 5 of 110) of GH-treated cancer survivors who had received cranial radiotherapy subsequently developed a SN. In contrast to the CCSS, the incidence of SN in the Mackenzie et al. study was not significantly greater in $\mathrm{GH}$-treated patients than in matched, non-GH-treated control patients $(2.7 \%, 3$ of $110 ; P=0.72)$. Notably, the median follow-up period in that study was 14.5 years, similar to that of the CCSS. More than $60 \%$ of the patients were adults when diagnosed with their primary cancer; however, among the childhood-onset subgroup, SNs occurred in five of $41 \mathrm{GH}$-treated patients and two of 42 non-GH-treated patients. Although the incidence of $\mathrm{SN}$ was 2.6-fold greater in the GH-treated patients, the difference was not statistically significant (28).

In both GeNeSIS and HypoCCS, as well as the CCSS $(18,19)$, meningiomas were the most common SN. The occurrence of three cases of osteosarcoma in GH-treated survivors of hematopoietic malignancies was considered a striking finding in the CCSS (19); no osteosarcomas were reported as SNs in GeNeSIS and HypoCCS. In all three studies, nearly all participants with a SN had a reported history of exposure to radiation as a consequence of treatment for their primary cancers. The primary cancers were frequently CNS malignancies or leukemia, which are known to be associated with an increased risk of SN $(29,30)$. Additionally, the time between primary cancer diagnosis and $\mathrm{SN}$ development was quite prolonged in GH-treated childhood cancer survivors, ranging from a median of 8.4 years in GeNeSIS to 20.3 years in HypoCCS 
participants. This timing is not inconsistent with that observed in the CCSS where the median time between primary cancer diagnosis and SN was 12.6 years (18). However, we note that childhood cancer survivors do not enroll into HypoCCS until after diagnosis of adult GHD, which may have prolonged the latency we observed.

There are limitations in the data described in this report. Neither GeNeSIS nor HypoCCS was specifically designed to evaluate the effect of GH treatment on the development of $\mathrm{SN}$ in childhood cancer survivors, as this information had not been published at the time of study design. As case report forms did not always contain details of the initial diagnosis and treatment of primary cancers, it is impossible to fully assess all the potential contributing factors to the development of the SN. Although broad search criteria were used to identify potential primary cancers and SNs, some cases may have been inadvertently omitted because of missing information. Potentially, some SN cases may have been omitted at the point of data inclusion for these analyses because of the open enrollment period and potential for loss to follow-up in observational research. In addition, as SNs may present many years after the primary childhood cancer and its treatment, the relatively short average duration of follow-up in these analyses limits their ability to capture all potential SN cases and limits the ability to interpret any relation of the observed SN to $\mathrm{GH}$ therapy. Another potential bias may be unknown differences between those patients who drop out of the study early vs those who are followed for longer; for example, there may be patients for whom concern of the risk for development of a SN or primary cancer recurrence increases. Data collection on $\mathrm{SNs}$ is as provided by the study investigators, the majority of which are endocrinologists and not oncologists, potentially leading to under-reporting of events. However, such potential may be mitigated by the vigilance for such outcomes, as stipulated in study protocols and product labeling, especially in childhood cancer survivors known to be at risk of SN. Furthermore, despite the large size of both GeNeSIS and HypoCCS databases, there were relatively few cases of childhood cancer survivors and even fewer SNs, further limiting our analyses. There were too few non-GH-treated childhood cancer survivors included from either database to draw meaningful conclusions about a potential increased risk of SN due to GH treatment. It is also difficult to assess the true risk of developing a $\mathrm{SN}$ from these databases, as a clinical bias is likely against starting $\mathrm{GH}$ in these patients, particularly adults, who have had a prior malignancy or disease with a worse prognosis.

In summary, the incidence of $\mathrm{SN}$ in both GeNeSIS and HypoCCS GH-treated childhood cancer survivors is consistent with the published literature, suggesting increased risk of $\mathrm{SN}$ in childhood cancer survivors who may be treated with GH. Longer observation is recommended to further clarify the extent of any risk.

\section{Declaration of interest}

W W Woodmansee is a former employee and stockholder of Eli Lilly and Company. C J Child, A G Zimmermann, Q Rong, and W F Blum are employees and shareholders of Eli Lilly and Company. E M Erfurth, L L Robison, P Beck-Peccoz, and W W Woodmansee are members of medical research advisory boards for, and have received consulting fees from Eli Lilly and Company. E M Erfurth has also received consulting fees from Pfizer.

\section{Funding}

This study was sponsored by Eli Lilly and Company. In compliance with the Uniform Requirements for Manuscripts, established by the International Committee of Medical Journal Editors, the sponsor of this study did not impose any impediment, directly or indirectly, on the publication of the study's results.

\section{Acknowledgements}

The authors express their gratitude to all GeNeSIS and HypoCCS patients, investigators, and study coordinators for their commitment to these observational studies. W W Woodmansee developed the initial draft of the manuscript, managed all subsequent drafts and had full access to primary study data related to this project. All coauthors were involved in manuscript development and were in agreement with the final version. The authors acknowledge the independent medical writing/editorial assistance provided by Rebecca Lew, PhD, of ProScribe Medical Communications (www.proscribe.com.au), funded by Eli Lilly and Company, who did not impose any restrictions, directly or indirectly, on the content of the manuscript. ProScribe's services complied with international guidelines for Good Publication Practice (GPP2).

\section{References}

1 Alvarez JA, Scully RE, Miller TL, Armstrong FD, Constine LS, Friedman DL \& Lipshultz SE. Long-term effects of treatments for childhood cancers. Current Opinion in Pediatrics 200719 23-31. (doi:10.1097/MOP.0b013e328013c89e)

2 Mulder RL, Kremer LC, van Santen HM, Ket JL, van Trotsenburg AS, Koning CC, Schouten-van Meeteren AY, Caron HN, Neggers SJ \& van Dalen EC. Prevalence and risk factors of radiation-induced growth hormone deficiency in childhood cancer survivors: a systematic review. Cancer Treatment Reviews 200935 616-632. (doi:10.1016/j.ctrv.2009.06.004)

3 Schmiegelow M, Lassen S, Poulsen HS, Feldt-Rasmussen U, Schmiegelow K, Hertz H \& Muller J. Cranial radiotherapy of childhood brain tumours: growth hormone deficiency and its relation to the biological effective dose of irradiation in a large population based study. Clinical Endocrinology 200053 191-197. (doi:10.1046/j.1365-2265.2000.01079.x)

4 Gurney JG, Ness KK, Sibley SD, O'Leary M, Dengel DR, Lee JM, Youngren NM, Glasser SP \& Baker KS. Metabolic syndrome and growth hormone deficiency in adult survivors of childhood acute lymphoblastic leukemia. Cancer $2006 \quad 107$ 1303-1312. (doi:10.1002/cncr.22120)

5 Clayton PE, Banerjee I, Murray PG \& Renehan AG. Growth hormone, the insulin-like growth factor axis, insulin and cancer risk. Nature Reviews. Endocrinology 20117 11-24. (doi:10.1038/ nrendo.2010.171)

6 Samani AA, Yakar S, LeRoith D \& Brodt P. The role of the IGF system in cancer growth and metastasis: overview and recent insights. Endocrine Reviews 200728 20-47. (doi:10.1210/er. 2006-0001)

7 Key TJ, Appleby PN, Reeves GK \& Roddam AW. Insulin-like growth factor 1 (IGF1), IGF binding protein 3 (IGFBP3), and breast cancer 
risk: pooled individual data analysis of 17 prospective studies. Lancet Oncology 201011 530-542. (doi:10.1016/S14702045(10)70095-4)

8 Rinaldi S, Cleveland R, Norat T, Biessy C, Rohrmann S, Linseisen J, Boeing H, Pischon T, Panico S, Agnoli C et al. Serum levels of IGF-I, IGFBP-3 and colorectal cancer risk: results from the EPIC cohort, plus a meta-analysis of prospective studies. International Journal of Cancer 2010126 1702-1715. (doi:10.1002/ijc.24927)

9 Roddam AW, Allen NE, Appleby P, Key TJ, Ferrucci L, Carter HB, Metter EJ, Chen C, Weiss NS, Fitzpatrick A et al. Insulin-like growth factors, their binding proteins, and prostate cancer risk: analysis of individual patient data from 12 prospective studies. Annals of Internal Medicine $2008 \mathbf{1 4 9} 461-471$.

10 Chung TT, Drake WM, Evanson J, Walker D, Plowman PN, Chew SL, Grossman AB, Besser GM \& Monson JP. Tumour surveillance imaging in patients with extrapituitary tumours receiving growth hormone replacement. Clinical Endocrinology 200563 274-279. (doi:10.1111/j.1365-2265.2005.02338.x)

11 Jostel A, Mukherjee A, Hulse PA \& Shalet SM. Adult growth hormone replacement therapy and neuroimaging surveillance in brain tumour survivors. Clinical Endocrinology 200562 698-705. (doi:10.1111/j.1365-2265.2005.02282.x)

12 Popovic V, Mattsson AF, Gaillard RC, Wilton P, KoltowskaHaggstrom M \& Ranke MB. Serum insulin-like growth factor I (IGF-I), IGF-binding proteins 2 and 3, and the risk for development of malignancies in adults with growth hormone $(\mathrm{GH})$ deficiency treated with GH: data from KIMS (Pfizer International Metabolic Database). Journal of Clinical Endocrinology and Metabolism 2010 95 4449-4454. (doi:10.1210/jc.2010-0287)

13 Svensson J, Bengtsson BA, Rosen T, Oden A \& Johannsson G. Malignant disease and cardiovascular morbidity in hypopituitary adults with or without growth hormone replacement therapy. Journal of Clinical Endocrinology and Metabolism 200489 3306-3312. (doi:10.1210/jc.2003-031601)

14 Swerdlow AJ, Higgins CD, Adlard P \& Preece MA. Risk of cancer in patients treated with human pituitary growth hormone in the UK, 1959-85: a cohort study. Lancet 2002360 273-277. (doi:10.1016/S0140-6736(02)09519-3)

15 Swerdlow AJ, Reddingius RE, Higgins CD, Spoudeas HA, Phipps K, Qiao Z, Ryder WD, Brada M, Hayward RD, Brook CG et al. Growth hormone treatment of children with brain tumors and risk of tumor recurrence. Journal of Clinical Endocrinology and Metabolism 200085 4444-4449. (doi:10.1210/jc.85.12.4444)

16 Wilton P, Mattsson AF \& Darendeliler F. Growth hormone treatment in children is not associated with an increase in the incidence of cancer: experience from KIGS (Pfizer International Growth Database). Journal of Pediatrics $2010 \quad 157$ 265-270. (doi:10.1016/j.jpeds.2010.02.028)

17 Child CJ, Zimmermann AG, Woodmansee WW, Green DM, Li JJ, Jung H, Erfurth EM \& Robison LL. Assessment of primary cancers in GH-treated adult hypopituitary patients: an analysis from the Hypopituitary Control and Complications Study. European Journal of Endocrinology 2011165 217-223. (doi:10.1530/EJE11-0286)

18 Ergun-Longmire B, Mertens AC, Mitby P, Qin J, Heller G, Shi W, Yasui Y, Robison LL \& Sklar CA. Growth hormone treatment and risk of second neoplasms in the childhood cancer survivor. Journal of Clinical Endocrinology and Metabolism $2006913494-3498$. (doi:10.1210/jc.2006-0656)
19 Sklar CA, Mertens AC, Mitby P, Occhiogrosso G, Qin J, Heller G, Yasui Y \& Robison LL. Risk of disease recurrence and second neoplasms in survivors of childhood cancer treated with growth hormone: a report from the Childhood Cancer Survivor Study. Journal of Clinical Endocrinology and Metabolism 200287 3136-3141. (doi:10.1210/jc.87.7.3136)

20 Webb SM, Strasburger CJ, Mo D, Hartman ML, Melmed S, Jung H, Blum WF \& Attanasio AF. Changing patterns of the adult growth hormone deficiency diagnosis documented in a decadelong global surveillance database. Journal of Clinical Endocrinology and Metabolism 200994 392-399. (doi:10.1210/jc.2008-0713)

21 Louis DN, Ohgaki H, Wiestler OD, Cavenee WK, Burger PC, Jouvet A, Scheithauer BW \& Kleihues P. The 2007 WHO classification of tumours of the central nervous system. Acta Neuropathologica 2007 114 97-109. (doi:10.1007/s00401-007-0243-4)

22 Blum W \& Schweizer R. Insulin-like growth factors and their binding proteins. In Diagnostics of Endocrine Function in Children and Adolescents, pp 166-199. Ed. M Ranke, Basel: S. Karger, 2003.

23 Blum WF \& Breier BH. Radioimmunoassays for IGFs and IGFBPs. Growth Regulation 19944 (Suppl 1) 11-19.

24 Boettcher C, Schaefer M, Wudy SA \& Blum WF. Cross-calibration of IGF-I and IGFBP-3 assays to obtain comparable values. Hormone Research $2006 \mathbf{6 5} 178$ (abstract).

25 Gaynor JJ, Feuer EJ, Tan CC, Wu DH, Little CR, Straus DJ, Clarkson BD \& Brennan MF. On the use of cause-specific failure and conditional failure probabilities: examples from clinical oncology data. Journal of the American Statistical Association 199388 400-409. (doi:10.1080/01621459.1993.10476289)

26 Inskip PD, Ries LAG, Cohen RJ \& Curtis RE. New malignancies following childhood cancer. In New Malignancies Among Cancer Survivors: SEER Cancer Registries, 1973-2000, pp 465-482. Eds RE Curtis, DM Freedman, E Ron, LAG Ries, DG Hacker, BK Edwards, MA Tucker \& JF Fraumeni, Bethesda: National Cancer Institute, NIH Publ. No. 05-5302, 2006.

27 Jazbec J, Ecimovic P \& Jereb B. Second neoplasms after treatment of childhood cancer in Slovenia. Pediatric Blood E Cancer 200442 574-581. (doi:10.1002/pbc.20025)

28 Mackenzie S, Craven T, Gattamaneni HR, Swindell R, Shalet SM \& Brabant G. Long-term safety of growth hormone replacement after CNS irradiation. Journal of Clinical Endocrinology and Metabolism 201196 2756-2761. (doi:10.1210/jc.2011-0112)

29 Hijiya N, Hudson MM, Lensing S, Zacher M, Onciu M, Behm FG, Razzouk BI, Ribeiro RC, Rubnitz JE, Sandlund JT et al. Cumulative incidence of secondary neoplasms as a first event after childhood acute lymphoblastic leukemia. Journal of the American Medical Association 2007297 1207-1215. (doi:10.1001/jama. 297.11.1207)

30 Neglia JP, Robison LL, Stovall M, Liu Y, Packer RJ, Hammond S, Yasui Y, Kasper CE, Mertens AC, Donaldson SS et al. New primary neoplasms of the central nervous system in survivors of childhood cancer: a report from the Childhood Cancer Survivor Study. Journal of the National Cancer Institute 200698 1528-1537. (doi:10.1093/jnci/djj411)

Received 2 November 2012

Revised version received 23 January 2013

Accepted 28 January 2013 